\title{
SULLO SPETTRO DELLE ONDE SISMICHE DESTATE DA ESPLOSIONI $\left(^{*}\right)$
}

\author{
H. MenzeL
}

Le leggi dello spettro di onde sismiche prodotte da esplosioni sono ancora relativamente poco studiate. Si sa che lo spettro d'onda Mintrop dipende molto dalla distanza del sismografo dal punto d'esplosione. A piccole distanze la frequenza dominante è altissima, spesso maggiore di $100 \mathrm{HZ}$, mentre a grandi diatanze la frequenza è di soli pochi $\mathrm{HZ}$. Nelle onde riflesse si osservano in generale, frequenze che in un ambito non troppo grande stanno intorno ai $50 \mathrm{HZ}$. Anche con queste onde si possono constatare mutamenti nello spettro con la distanza e la profondita degli estratti riflettenti. Da questi dati di esperienze consegue che non solo il meccanismo all'atto della esplosione, ma anche quello del propagarsi delle onde nella terra influiscono sulla formazione dello spettro delle onde. Le considerazioni seguenti si riferiscono essenzialmente ad alcuni effetti, che hanno per conseguenza dei cambiamenti dello spettro di onde sismiche dovute ad esplosioni. Si tratterà però anche della formazione dello spettro primario, conseguente all'esplosione.

Quando si ricercano i fenomeni che determinano un cambiamento dello spettro delle onde sismiche ci si trova imbarazzato non per mancanza di tali effetti, ma perché ce ne sono troppi. Ben nota è sotto questo aspetto, la parte che ha la differenziazione del suolo percorso dall'onda da un mezzo puramente elastico. Ma non si vogliono discutere qui le particolarità. Nell'ambito di questo lavoro, la crosta terrestre verrà trattata come mezzo puramente elastico. Con questo, non si vuole certamente affermare, che la viscosità, l'attrito interno o l'elasticità susseguente non siano senza effetto o solo di poco effetto. Scopo di questa indagine non è la completa soluzione del problema della frequenza ma la preparazione della soluzione stessa.

Qui si tratteranno solo quegli effetti che in un mezzo puramente elastico determinano un cambiamento dello spettro d'onda. Si tratta essenzialmente di due fenomeni fisici distinti : 1) interferenza; 2) dispersione di energia per riflessione, rifrazione, e flessione.

L'importanza dell'interferenza per la formazione dello spettro risulta dalla circostanza che le esplosioni vengono eseguite alcuni metri al di sotto della superficie terrestre. Interferiscono allora nel sottosuolo più profondo due unde: l'onda Irodotta direttamente dall'esplosione e l'nnda riflessa alla superficie. A secouda del diverso tragitto temporale di queste due onde in un determinatc punto, si intensificheranno ivi determinati campi di frequenza dello spettro, altri si indeboliranno. Supponendo che il punto d'esplosione si trovi a $15 \mathrm{~m}$ sotto la superficie terrestre e che la velocità d'onda nello strato superiore della terra sia di $1500 \mathrm{~m} / \mathrm{s}$, la differenza di tragitto in un punto che si trovi perpendicolarmente sotto al centro d'esplosione, importa $20 \mathrm{~ms}$. Nello spettro d'onda il campo di frequenza verrà quindi rafforzato di $50 \mathrm{HZ}$. Ora si deve calcolare la differenza di tragitto in

(") Traduzione in lingua italiana della Nota di pag. 301. 
un punto $P$ avente dall'epicentro la distanza $x$ (v. fig. 1). Il piano riflettente si trovi alla profondità $H$ e sia parallela alla superficie terrestre. $S$ sia il centro di esplosione $S$ l'immagine di $S, B$ quella di $S$, entrambi rispetto al piano riflettente nel fondo. Se è $v$ la velocità d'onda, si calcola la diversità del tragitto in $P$ secondo la formula

$$
\Theta=\frac{8 H d}{l_{1}+l_{2}} \frac{1}{v}=4 \frac{I}{l} \frac{\vec{J}}{v}
$$

dove $l$ è la media di $l_{1}$ e $l_{\underline{\underline{a}}}$. Dall'equazione [1] risulta:

1) essendo fisso $H$ e $x$ il campo di frequenza rafforzato dall'interferenza si sposta nello spettro verso valori più piccoli, se la profondità del punto di esplosione aumenta.

2) Essendo fisso $d$ e $x$ il campo di frequenza rafforzato si trova presso valori più piccoli, se la superficie riflettente si trova più in basso.

3) Essendo fisso $H$ e $d$ il campo di frequenza rafforzato si sposta verso valori maggiori, se $x$ cresce.

In senso puramente qualitativo questi tre fenomeni si possono effeftivamente constatare nel caso di osservazioni sismiche per esplosioni. Si è iniziato un esame quantitativo, che soltanto può decidere sul valore o meno di una teoria. Certamente la [1] è ancora troppo grossolana per poter restituire quantitativamente le effettive condizioni.

L'accenno che il campo di frequenza si rafforza per interferenza di circa $50 \mathrm{~Hz}$, non basta a spiegare il predominare di queste frequenze nelle onde riflesse. Precisamente, se questo campo di frequenza ha nello spettro primario una parte subordinata, non si capisce facilmente come possa essere tanto rafforzato dall'interferenza, da dominare alla fine. E dunque importante fare alcune considerazioni sulla distribuzione delle frequenze nello spettro primario. Per tale assunto offre lo spunto migliore un lavoro di $\mathrm{H}$. Morris di recente pubblicazione. Nel corso dei procedimenti Morris distingue tre fasi dopo l'avvenuta esplosione. Nella primo fase brevissima, l'esplosivo si trasforma in un gas caldo ad alta tensione, senza che le pareti del foro di tiro cedano notevolmente. Nel secondo stadio si diffonde un'onda d'urto a forma di sfera, mentre precipitano le pietre nel mezzo fino a raggiungere un raggio critico $R$. Questo è caratterizzato dal fatto che limporto massimale della Tensione elastica prodotta nella roccia dall'onda d'urto è tanto diminuito, che il successivo avvenimento, cioè il terzo stadio, decorre secondo le leggi della Teoria dell'elasticità. Questo terzo stadio sarà qui considerato meglio. In un mezzo elastico omogeneo, isotropo illimitato, in tutte le direzioni, sia presente una cavità a forma di sfera di raggio $R$. Sulla superficie della sfera sia esercitata una pressione dall'interno, secondo una qualsiasi legge di tempo $\Phi(t)$. Si deve calcolare l'onda elastica che origina alla superficie della sfera.

Per motivi di simmetria è possibile solo un'onda longitudinale a forma sferica. Ponendo l'origine di un sistema spaziale di coordinate polari nel centro della sfera cava, la soluzione del problema dipende solo da una coordinata spaziale, la lunghezza $r$ del raggio vettore, e il vettore $\sigma$ dello spostamento elastico ha solo una componente $\vec{\sigma}$ in direzione del raggio vettore. $\dot{E}$ 
cioè

$$
\vec{\sigma}=\operatorname{grad} F\left(r_{1} t\right)
$$

$$
\sigma r=\frac{\delta F}{\delta r}
$$

Dall'equazione della teoria dell'elasticità

$$
\varrho \frac{\delta^{2} \sigma}{\delta t^{2}}=(\lambda+2 \mu) \operatorname{grad} \operatorname{div} \vec{\sigma}-\mu \operatorname{rot} \operatorname{rot} \vec{\sigma}
$$

segue poi per $F$ l'equazione differenziale

$$
\varrho \frac{\delta^{2} F}{\delta t^{2}}=(\lambda+2 \mu) \Delta F
$$

Si indicano con $\varrho$ la densità del mezzo, e $\lambda$ e $\mu$ sono le sue costanti di Lamé. La componente normale del tensore di tensione si calcola con

$$
N=(\lambda+2 \mu) \operatorname{div} \overrightarrow{\sigma-4} \mu \frac{1}{r} \sigma_{r}
$$

ne consegue:

$$
N=(\lambda+2 \mu) \Delta F-4 \mu \frac{1}{r} \frac{\delta F}{\delta r}
$$

All'equazione differenziale [3 a] s'aggiunge ancora la condizione al contorno

$$
(\lambda+2 \mu) \Delta F-4 u \frac{l}{R} \frac{\delta F}{\delta r}+\Phi(t)=0 \quad \text { per } r=R
$$

Da [3 a] consegue la soluzione

$$
F=\frac{1}{r} H(r-v t)
$$

dove

$$
v=\sqrt{\frac{\overline{\lambda+2 u}}{\varrho}}
$$

e $H$ è una funzione ancora arbitraria. Da [6] e [5] si ottiene

$$
\frac{\lambda+2 \mu}{R} H^{\prime \prime}(R-v t)-\frac{4 \mu}{R^{2}} H^{\prime}(R-v t)+\frac{4 \mu}{R^{3}} H(R-v t)+\Phi(t)=0
$$

Se si pone $R-v t=\xi$ e $\Phi(t)=\psi(\xi)$, si può determinare $H$ dalla consueta equazione differenziale 


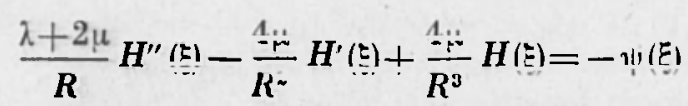

La soluzione è

$\left.H(r-v t)=\frac{i}{4} \frac{R^{2}}{\varrho v} \frac{\lambda+2 \mu}{\sqrt{\mu(\lambda+\mu)_{0}}} \int e_{0}^{\sigma_{1}\left(t-\frac{r-R}{v}-\tau\right)}-e^{\sigma_{1}\left(t-\frac{r-R}{v}-\tau\right)}\right]_{[9]} \Phi(\tau) d \tau$

Le grandezze $\sigma_{1}$ e $\sigma_{2}$ si calcolano

$$
\sigma_{1}=\frac{v}{R}\left(-\frac{2 \mu}{\lambda+2 \mu}+2 i \frac{\sqrt{\mu(\lambda+\mu)}}{\lambda+2 \mu}\right), r_{i}=\frac{v}{\kappa}\left(-\frac{2 \mu}{\lambda+2 \mu}-2 i \frac{\sqrt{\mu(\lambda+\mu)}}{\lambda+2 \mu}\right.
$$

L'apparire della funzione $e$ con argomento complesso uella funzione integrale alla [9], significa che il movimento del suolo contiene in ogni punto una parte, che può essere designata come oscillazione propria smorzala. La frequenza di questa oscillazione propria $\dot{e}$

$$
n=2 \frac{\because}{R} \sqrt{\frac{\mu(\hat{\mu}+\mu)}{\lambda+2 u}}
$$

e la sua costante di smorzamento

$$
\varepsilon=2 \frac{v}{R} \frac{\mu}{\lambda+2 u}
$$

Per meglio studiare l'equazione [9], si deve conoscere la funzione $\Phi(t)$. Purtroppo, non si sa ancora nulla di preciso circa questa funzione. Riteniamo di non essere molto discosti dalla realtà, se si calcola con l'ipotesi

$$
\begin{array}{lll}
\Phi(t)=0 & \text { per } & -\infty<t<0 \\
\Phi(t)=a t \mathrm{e}^{-\alpha t} & & 0 \leq t<\infty \quad, \quad u>0
\end{array}
$$

Dalle [12], [9], [6] e [22 a] segue poi

$$
\begin{gathered}
\sigma_{\mathrm{r}}=\frac{a R^{2}}{2 \sqrt{u(\lambda+u)\left[(\alpha-\varepsilon)^{2}+\omega^{2}\right]^{2}}} \frac{1}{r} \\
\left\{\left(\frac{v}{r}-\varepsilon\right)\left[(\alpha-\varepsilon)^{2}-\omega^{2}\right]+2 \omega^{2}(\alpha-\varepsilon)\right\} e^{-\varepsilon\left(t-\frac{r-k}{v}\right)} \sin \omega\left(t-\frac{r-R}{v}\right) \\
+\omega\left[(\alpha-\varepsilon)^{2}-\omega^{2}-2\left(\frac{\ddot{v}}{r}-\varepsilon\right)(\alpha-\varepsilon)\right] e^{-\varepsilon\left(t-\frac{r \cdots K}{v}\right)} \cos \omega\left(t-\frac{r-R}{v}\right)
\end{gathered}
$$




$$
\begin{aligned}
& \left.-\left\{\left(\frac{v}{r}-\varepsilon\right)\left[(\alpha-\varepsilon)^{2}-\omega^{2}\right]+2(1)^{2}(\alpha-\varepsilon)\right\} \omega\left(t-\frac{r-R}{v}\right)\right] e^{-\alpha\left(t-\frac{r-R}{v}\right)} \\
& -\omega\left[(\alpha-\varepsilon)^{2}-\omega^{2}-2\left(\frac{n}{r}-\varepsilon\right)((l-\varepsilon)]\left[l+(\alpha-\varepsilon)\left(t-\frac{r-R}{v}\right)\right] e^{-c\left(t-\frac{r-R}{v}\right)}\right.
\end{aligned}
$$

I due primi termini dell'equazione [13] rappresentano loscillazione propria smorzata, che si propaga nel mezzo, i due termini rappresentano la propagazione della perturbazione. Ampiezza e fase dell'oscillazione propria sono dipendenti da $r$. Perciò l'immagine dell'oscillazione muta da punto a punto. Se vale $r>>R$, il decorso dell'oscillazione è spazialmente quasi costante.

Il calcolo numerico dell'equazione [13] richiede delle ipotesi circa $\lambda, \mu, r$, $R$, $\alpha$ et $a$. Approssimativamente si può porre $\lambda=\mu$. Per la velocità nel diluviale si può porre, con qualche giustificazione, $v=1,6 \mathrm{~m} / \mathrm{ms}$. La grandezza $R$ dipende dalla quantità di esplosivo. E difficile per questo valore raggiungere una stima sicura, perché ne sappiamo ancora troppo poco. In condizioni normali, $R$ si trova in un ambito di valori da alcuni $\mathrm{dem}$ fino a pochi $\mathrm{m}$. Si supponga qui $R=1 \mathrm{~m}$. La ricerca allora

$$
()=1,51 \mathrm{~ms}^{-1}
$$

cioè

$$
v=\frac{\omega}{2 \pi}=240 H_{z}
$$

$$
\varepsilon=1,067 \mathrm{~ms}^{-1}
$$

Data la poca sicurezza nell'ipotesi di $R$, (i) et $\varepsilon$ possono assumere valori metà o doppi di quelli riportati. Il rapporto (1) $\varepsilon$ non risente di questa incertezza e vale

$$
\frac{\omega}{\varepsilon}=\sqrt{ } ?
$$

nell'ipotesi suddetta. Il decremento logaritmico vale dunque

$$
\delta=4,44
$$

Ancor più incerta che nei riguardi di $R$ è azzardare un'ipotesi per $\alpha$. Dal lavoro già citato di Morris, si può dedurre che $\alpha$ potrebbe essere della stessa grandezza di $\varepsilon$.

Poiché per il caso di $a=\varepsilon$ la formula [13] diventa particolarmente semplice, si deve anzitutto calcolare con questa ipotesi. La grandezza non ha importanza per gli scopi di questa ricerca, perché non ha influenza sulla forma delle oscillazioni. Si supponga ancora $r>R$ e si ponga $t-\frac{r}{v}=t^{\prime}$. Risulta allora dalla [13] il movimento del suolo. 


$$
\sigma_{\mathrm{r}}=\frac{a R^{*}}{2 \sqrt{u(\lambda+\mu)} \omega} \frac{e^{-\varepsilon t^{\prime}}}{r}\left[\gamma^{\prime} \overline{1, j} \sin \left(\omega \boldsymbol{t}^{\prime}-0,96\right)+1-\varepsilon \boldsymbol{t}^{\prime}\right]
$$

nella figura 2 questa oscillazione è rappresentata dalla linea intera. La linea tratteggiata dà un'immagine dell'andamento nel tempo dello impulso di perturbazione $\Phi(t)=a \imath e^{-\alpha t}$

Per tener conto dellincertezza nellipotesi di $\alpha$, consideriamo ancora l'estremo caso limite $\alpha>>$. In questo caso da [13] deriva:

$$
\sigma_{\mathbf{r}}=-\frac{a R^{2} \omega}{2 \sqrt{\mu(\lambda+\mu)} \alpha^{2}} \frac{e^{-\varepsilon t^{\prime}}}{r}\left[\gamma^{\prime} 1,5 \sin \left(\omega t^{\prime}-0,96\right)+e^{--\alpha t^{\prime}}\left(1+\alpha t^{\prime}\right)\right]
$$

Il secondo termine della parentesi quadra di [15 a] è notevole solo per valori estremamente piccoli di $\boldsymbol{t}^{\prime}$. Prescindendo dalla primissima parte della curva, [1 $a$ a] rappresenta la pura oscillazione propria. La trattazione dell'altro caso limite $\alpha<<\varepsilon$ non $\dot{e}$ necessaria. $\mathrm{E}$ improbabile che l'impulso di perturbazione nel raggio critico $R$ sia già diviso su molti.

Dalla figura 2 e dalla equazione [15] e [15 a] risulta che, la frequenza prupria $v=\frac{\omega}{\overline{2} \pi}$ nello spettro primario dell'onda di esplosione deve predominare. Questa frequenza importa secondo lipotesi qui fatte $240 \mathrm{OHZ}$. Certamente queste ipotesi non sono ben fondate; c possibile modificarle fino a ridurre la frequenza propria sotto ai $100 \mathrm{HZ}$. Gli spettri osservati nelle registrazioni delle esplosioni si formano quindi probabilmente in seguito a una modificazione sostanziale dello spettro primario. Si può dubitare che solo l'effetto d'interferenza discusso procedentemente possa determinare questo cambiamento dello spettro primario. Col rapido svanire del movimento, secondo la [15] ci si può appena immaginare come possa aver luogo questo effetto d'interferenza per le normali profondità dei !oci di scoppio. Devono apparire ancora altri effetti, che in primo luogo attenuano in modo particolarmente sensibile le alte frequenze, e in secondo luogo hanno per conseguenza un prolungamento dellintero impulso.

Clewell e Simon hanno ${ }^{(2)}$ recentemente pubblicato un lavoro in cui si sostiene l'opinione che la crosta terrestre agisca come un filtro passa bande. L'affievolirsi delle frequenze profonde dev'essere un effetto d'interferenza, che subentra quando la lunghezza d'onda è grande rispetto allo spessore degli strati, racchiusi nel mezzo omogeneo, dal quale differiscono per densità e costanti elastiche. Secondo l'avviso di questi autori, l'estinguersi delle alte frequenze, prescindendo dall'azione dolla viscosità, viene determinata dalla dispersione su piccole disomogeneità, come per la diffusione della luce nella teoria di Rayleigh. Della presenza di tali strati sottili e di disomogeneità non si può dubitare. Una valutazione quantitativa del loro influsso su onde attraversanti è oltremodo difficile. Per il caso di strato nel mezzo si può tentare una valutazione con la premessa - certamente molto specifica che la normale dell'onda sia perpendicolare alle superfici limiti dello strato, supposte parallele. 
Il punto zero del sistema di Coordinate sia posto nel piano superiore di delimitazione dello strato inserito ( $v$. fig. 3).

L'asse $z$ sia diretto perpendicolarmente verso il basso; $d$ sia lo spessore dello strato. Al di sopra e al di sotto dello strato sia $S_{1}$ la densità, $\lambda_{1}$ e $\mu_{1}$ le costanti di Larve. I corrispondenti valori nello strato siano $\lambda_{\ldots,}$ e $\mu_{\%}$. Perpendicolarmente al piano $z=O$, incida un'onda piana $l$ longitudinale. Nel piano di incidenza ha luogo una divisione dell'energia d'onda. Una parte dell'energir viene riflessa nel mezzo superiore, un'altra parte penetra dentro allo strato. Nel piano $z=d$ ha luogo lo stesso processo. La parte di energia riflessa da questa superficie investe dal basso il piano $z=O$, dove ha nuovamente luogo una divisione di energia. Il processo si ripete ininterrottamente. Da ciascuna delle due superfici limiti viene dunque irradiata verso l'alto e verso il basso una serie infinita convergente di onde, che interferiscono fra loro. L'onda incidente sia rappresentata da

$$
w_{1}=A_{1} e^{i \omega\left(\iota z a_{1}\right)}
$$

dove $w_{1}$ è lo spostamento (in direzione $z$ ) e $a_{1}$ è la velocità nel mezzo superiore. Dalla superficie $Z=O$ viene irradiata verso l'alto l'onda

$$
\bar{w}_{1}=B_{1} e^{i(1)\left(t+z_{i} a_{1}\right)}
$$

e verso il basso nello strato l'onda:

$$
w_{2}=A_{2} e^{i c\left(t-z a_{t}\right)}
$$

corrispondentemente dal piano $z=d$ origina nello strato l'onda:

$$
\bar{w}_{2}=B . e^{i \omega\left(t+z / a_{y}\right)}
$$

e verso il basso l'onda

$$
w_{3}=A_{3} e^{i a\left(t-z / a_{1}\right)}
$$

$\bar{w}_{1}, \bar{w}_{\text {, }}$ et $w_{3}$ sono onde che risultano dall'interferenza descritta. Vale

$$
a_{v}=\int_{y}^{\sqrt{\lambda_{\mathrm{v}}+2 \eta_{\mathrm{v}}}} \quad(v=1,2)
$$

e le costanti $B_{1}, A_{0}, B_{0}, A_{3}$ si calcolano dalle condizioni al contorno per $z=O$ e $Z=d$. Le condizioni al contorno richiedono costanza degli spostamenti e delle componenti normali della tensione. Si trova il sistema di equazione:

$$
\begin{aligned}
& \begin{array}{llll}
A_{1} & +B_{1}= & A_{0} & +B_{2}
\end{array} \\
& \varrho_{1} a_{1}\left(A_{1} \quad-B_{1}\right)=\quad \varrho_{2} a_{2}\left(A_{2} \quad-B_{2}\right) \\
& A_{0} e^{-i \frac{\omega d}{a_{2}}}+B_{0} e^{+i \frac{\omega d}{a_{2}}}=A_{3} e^{-i \frac{\omega d}{\bar{z}_{1}}} \\
& \mathrm{C}_{2} a_{2}\left(A_{2} e^{-i \frac{\omega) d}{c_{2}}}-B_{2} e^{+i \frac{\omega d}{\sigma_{2}}}=\varrho_{1} a_{1} A_{3} e^{-i \frac{\omega d}{a_{1}}}\right.
\end{aligned}
$$


se si pone per abbreviare:

$$
\frac{\varrho_{0} a_{0}}{\varrho_{1} a_{1}}=p
$$

si ottiene così la soluzione:

$$
\begin{aligned}
& B_{1}=\frac{i\left(\frac{1}{p}-p\right) \sin \frac{\omega d}{a_{2}}}{2 \cos \frac{\omega d}{a_{0}}+\left(\frac{1}{p}+p\right) \sin \frac{\omega d}{a_{2}}} A_{1} \\
& A_{3}=\frac{2 e^{i \frac{\omega d}{u_{1}}}}{2 \cos \frac{\omega d}{a_{0}}+i\left(\frac{1}{\left(\frac{1}{p}+p\right) \sin \frac{\omega d}{a_{2}}} A_{1}\right.}
\end{aligned}
$$

Le costanti $A_{2}$ e $B_{2}$ qui non interessano.

Le espressioni da [16] a [16 d] non rappresentano ancora di per sé onde reali, giacché esse rappresentano in ogni luogo $z$ una oscillazione persistente da $t=-\infty \quad t=\infty$. Per giungere a onde reali si devono considerare le grandezze $A_{1}, A_{2}, A_{3}, B_{1}$ e $B_{2}$ come funzioni di $\omega$ e integrare le parti destre delle equazioni da [16] e [16 a] per is da $-\infty a+\infty$. Da ciò risulta:

$$
\begin{aligned}
& w_{1}=\int_{-\infty}^{+\infty} A(\omega) e^{i\left(t\left(t-z_{1} a_{1}\right)\right.} d \omega \\
& \bar{w}_{1}=\int_{-\infty}^{+\infty} A(\omega) \frac{i\left(\frac{l}{p}-p\right) \sin \frac{\omega d}{a^{2}}}{2 \cos \frac{\omega d}{a_{2}}+\imath\left(\frac{l}{l p}+p\right) \sin \frac{\omega d}{a_{2}}} e^{i \omega\left(t+z / a_{1}\right)} d( \\
& w_{3}=\int_{-\infty}^{+\infty} A(\omega) \frac{2 e^{i \frac{(1) d}{a_{1}}}}{2 \cos \frac{(\omega d}{a_{2}}+i\left(\frac{l}{p}+p\right) \sin \frac{\omega d}{a_{2}}} e^{\left.i m(t) / \omega_{1}\right)} d \omega
\end{aligned}
$$

Sull'onda incidente si faccia la seguente ipotesi speriale:

$$
\begin{array}{lll}
w_{1}=o & \text { per } & t<z a_{1} \\
w_{1}=e^{i \sigma\left(t-z a_{1}\right)} & & t \geqslant z / a_{1}
\end{array}
$$


Sia o complesso con parte immaginaria positiva, e delle funzioni possa essere fisicamente reale solo la parte immaginaria.

La funzione $A((1))$ nelle equazioni [21 a] - [21 e] suona allora :

$$
A(\omega)=\frac{1}{2 \pi i} \frac{1}{\omega-\sigma}
$$

Integrando si puó calcolare orn

$$
w_{3}=\frac{l}{\pi i} \int_{-\infty}^{+\infty} \frac{e^{i \omega\left(t-\frac{z-d}{a_{1}}\right)}}{2 \cos \frac{(i)}{a_{n}}+i\left(\frac{1}{p}+p\right)} \frac{d(1)}{\sin \frac{\omega d}{a_{2}}} \frac{d-\sigma}{\omega-\sigma}
$$

I poli della funzione integranda si trovano per

$$
\omega=\sigma
$$

e per:

$$
\omega_{n}=\frac{a_{w}}{d} \pi n+i \frac{a_{o}}{d} \operatorname{arctgh} \frac{2}{p+1 / p} \quad(n=0, \pm 1 \pm 2 \ldots)
$$

Hanno dunque tutte una parte immaginaria positiva. Ne deriva:

$$
\begin{aligned}
& v_{3}=o \quad, \quad \text { per } t<\frac{z-d}{a_{t}}
\end{aligned}
$$

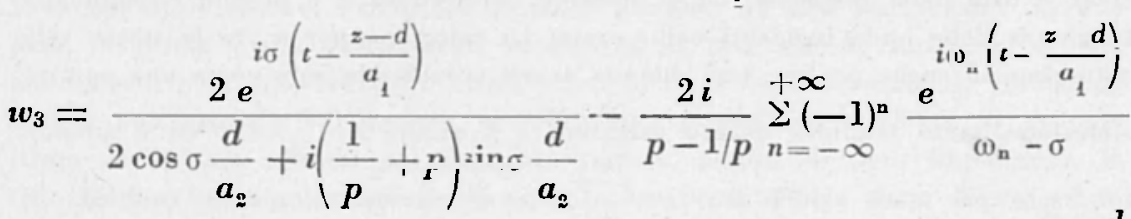

$$
\begin{aligned}
& \text { per } t=\frac{z-d}{a_{1}}
\end{aligned}
$$

In questo computo è stato supposto $\sigma \neq$ Non è difficile trattare anche il caso in cui un qualche $\omega_{n}$ coincida con $\sigma$. Ma qui lo si omette.

In modo del tutto analogo si deriva il valore dell'integrale da [21 b]. Si scrive anzitulto:

$$
\overline{\boldsymbol{u}}_{1}=\frac{1 / p-p}{4 \pi i} \int_{-\infty}^{+\infty} \frac{e^{i \omega\left(t+\bar{s}_{i}+a_{i}+d_{i}^{\prime} \omega_{0}\right)}}{2 \cos \frac{\omega) d}{a_{2}}+i\left(\frac{1}{p}+p\right) \sin \frac{\omega d}{a_{2}}} \frac{d \omega}{\omega-\sigma}
$$




$$
-\int_{-\infty}^{+\infty} \frac{e^{i \omega\left(t+z a_{i}+d / a_{o}\right)}}{2 \cos \frac{\omega d}{a_{o}}+i\left(\frac{1}{p}+p\right) \sin \frac{\omega d}{a_{o}}} \frac{d \omega}{\omega-\sigma}
$$

I poli sono gli stessi come nell'integrale di [2l c]

L'integrazione dà :

$$
\begin{aligned}
\bar{w}_{1}= & \frac{1}{2} \frac{\frac{1}{p}-p}{2 \cos \sigma \frac{d}{a_{2}}+i\left(\frac{1}{p}-p\right) \sin \sigma \frac{d}{a_{2}}}\left[e^{i \sigma\left(t+z / a_{1}+d / a_{2}\right)}-e^{i \sigma\left(t+z / a_{1}-d / a \cdot\right)}\right] \\
& +\frac{i}{\bar{z}} \sum_{n=-\infty}^{+\infty} \frac{(-1)^{\mathrm{n}}}{\omega_{\mathrm{n}}-\sigma}\left|e^{i\left(\omega_{\mathrm{n}}\left(t+z / a_{1}-d / a_{n}\right)\right.}-e^{i()_{\mathrm{n}}\left(t+z / a_{1}-d / a_{2}\right)}\right|
\end{aligned}
$$

Considerando più da vicino queste soluzioni si riconosce subito che contengono delle incongruenze fisiche, che derivano dalla declinazione contenuta nell'impostazione matematica. Così l'onda $u_{3}$ del posto $z=d$ si propagherebbe verso il hasso già al tempo $t=O$. Questo è naturalmente impossibile se l'onda incidente al tempo $t=O$ colpisce il luogo $z=O$. Tale incongruenza è una conseguenza del fatto che nell'ipotesi iniziale tutte le onde sono state supposte simultanee. Una giustificazione è data dalla premessa che lo spessore dello strato $d$ è piccolo rispetto alla lunghezza delle onde incidenti nello strato. La soluzione per $w$, e lo stesso vale naturalmente anche per $w_{1}$, può dunque essere considerata solo come una approssimazione, tanto migliore quanto maggiore è il valore $t-\frac{z-d}{a_{1}}$; ed è attendibile solo per quei valori di $t-\frac{z-d}{a_{1}}$ che importano un piccolo multiplo di $d / a_{2}$. Nel caso dell'onda $\bar{w}_{1}$ le onde rappresentate dai primi termini nelle parentesi quadre di [26] diventano al tempo $t=-\frac{z}{a_{1}}-\frac{d}{a_{2}}$, e le onde rappresentate dai secondi termini di queste parentisi solo al tempo $t=-\frac{z}{a_{1}}+\frac{d}{a_{2}}$. In seguito alla limitazione dell'ambito di velocità della soluzione ora esposta si possono senz'altro sovrapporre le due onde e si ottiene:

$$
\bar{w}_{1}=\frac{i\left(\frac{1}{p}-p\right) \sin \left(\frac{d}{\bar{u}_{2}}\right.}{2 \cos \sigma \frac{d}{a_{2}}+i\left(\frac{1}{p}+p\right) \sin \sigma \frac{d}{a_{2}}} e^{i \sigma\left(t+z / a_{1}\right)}
$$




$$
\underset{n=-\infty}{+\infty} \frac{(-1)}{\omega_{n}-\sigma} e^{n i \omega_{n}\left(\ell+z / a_{1}\right)} \sin \omega_{n} \frac{d}{a_{2}}
$$

Le onde $w_{3}$ e $\bar{w}_{1}$ constano di 3 parti. La prima ha la forma dell'onda incidente, con ampiezza e fase mutata. La seconda parte è aperiodica $(n=O)$, la terza $\dot{e}$ un'onda smorzata con periodo fondamentale $2 \frac{d}{a_{2}}$.

Le grandezze $\omega_{n}$ si possono considerare come frequenze proprie complesse dello strato eccitato dell'onda incidente. Essendo però la parte reale di $\omega_{\mathrm{p}}$ molto grande, l'onda con periodo fondamentale non avrà per lo meno per $2 \frac{d}{u_{2}}$, alcuna parte sostanziale. Per $\bar{w}_{1}$, dato il lieve ammontare assoluto di $\sigma \frac{d}{a_{2}}$ la prima parte può certamente perdere di importanza. L'effetto dello stato consisterà dunque nel fatto che l'onda rifratta viene mutita solo lievemente nella sua forma. Nell'onda riflessa dello strato le frequenze proprie dello strato possono eventualmente avere un loro ruolo. Nella figura 4 sono rappresentate a illustrazione di quanto fu detto. londa incidente e l'onda rifratta per un caso speciale.

Le ipotesi numeriche sono:

$$
\begin{aligned}
& a_{1}=1,6 \mathrm{~m} / \mathrm{ms} \quad ; \quad a_{2} 2,4 \mathrm{~m} / \mathrm{ms} ; p=1,5 ; d=1 \mathrm{~m} \\
& \sigma=(1.51+1,07 i) \mathrm{ms}^{-1}
\end{aligned}
$$

Quando gli spessori dello strato sono molto piccoli non è quindi da attendersi, per lo meno per l'incidenza perpendicolare delle onde, alcun mutamento molto notevole dello spettro. Nel caso limite opposto di uno spessore di strato molto grande rispetto alla lunghezza delle onde, non si può in generale ammettere alcun cambiamento dello speltro. Prima che si possa giungere ad una interferenza tra due onde la prima è già praticamente scomparsa. Il caso che la lunghezza d'onda nello strato e lo spessore dello strato siano di circa eguale ordine di grandezza, non si può esaurire col metodo precedentemente applicato. L'effetto di un tale strato su un'onda rifratta qui non sarà trattato, perché di poca importanza. Il caso invece che la potenza dello strato e la lunghezza d'onda siano di un ordine di grandezza pressoché eguale, è d'importanza per l'incidenza di un'onda sulla superficie terrestre. Supponiamo che la velocità dell'onda nel cosi detto strato di materiali sciolti sia di $0,8 \mathrm{~m} / \mathrm{ms}$. Un'onda nella quale predomini una frequenza di $240 \mathrm{HZ}$, avrà in questo strato una lunghezza d'onda di m 3,33 . É noto che lo spessore dello strato di sfaldamento è di eguale volume di grandezza. Si deve perciò considerare ora la teoria della riflessione per un'onda incidente perpendicolarmente alla superficie terrestre stratificata.

Il piano $z=O$ sia la superficie terrestre, e il piano $z=d$ sia il limite inferiore dello strato di materiali sciolti, che supporremo omogeneo e spessore costante. Siano $\varrho_{0} a_{0} e_{1} a_{1}$ densità e le velocità d'onda nello strato di materiali sciolti e nel fondo omogeneo rispettivamente. Sulla superficie $z=d$ incida al tempo $t=O$ l'onda $u_{1}$ dal basso. Essa viene scissa in un'onda riflessa $\bar{w}_{1} \mathrm{e}$ in una rifratta $w_{0}$. Al tempo $t=\frac{d}{a_{0}}, w_{1}$ batte sulla superficie $z=O$. L'onda riflessa 
da questa superficie sia $w_{0}$, e al tempo $t=\frac{2 d}{a_{0}}$ batte sulla superficie limite inferiore. Siano rispettivamente $w_{0}{ }^{(1)}$ e $\bar{w}_{1}(1)$ le onde generate per riflessione e rifrazione. Questi processi si ripetono all'infinito, ma l'energia delle onde parziali, dopo un numero finito di riflessioni, si rende praticamente trascurabile. Consideriamo anzitutto ciò che avviene sul piano $z=O$. Sia $\zeta_{0}$ l'oscillazione da osservarsi da questa superficie, in virtù dell'interferenza delle singole onde parziali: allora è:

$$
\begin{array}{cc}
\zeta_{0}=0 & \text { per }-c<t<\frac{d}{a_{0}} \\
\zeta_{0}=\left[w_{0}+\bar{w}_{0}\right]_{x}=0 & \text { per } \frac{d}{a_{0}}<t<3 \frac{d}{a_{0}} \\
\tau_{0}=\left[w_{0}+\bar{w}_{0}+\sum_{v=1}^{n}\left(w^{(v)}+\bar{w}_{0}^{(v)}\right)\right]_{x}=0 & \text { per } \left.(2 n+1) \frac{d}{a_{0}}<1<12 n+3\right) \frac{d}{a_{0}}
\end{array}
$$

In corrispondenza, si costruisca anche l'onda $W_{1}$ riflessa sul fondo, come sovrapposizione di onde parziali.

$$
\begin{array}{ll}
W_{1}=0 & -\infty<t-\frac{z-d}{a_{1}}<0 \\
W_{1}=\bar{w}_{1} & 0 \leq t-\frac{z-d}{a_{1}}<2 \frac{d}{a_{i j}} \\
W_{1}=\bar{w}_{1}+\bar{w}_{1}^{(1)} & 2 \frac{d}{a_{s}} \leq t-\frac{z-d}{a_{1}}<4 \frac{d}{a_{1}} \\
W_{1}=\bar{w}_{1}+\sum_{v=1}^{\mathrm{n} \bar{w}_{1}(v)} & 2 n \frac{d}{a_{i}}<t-\frac{z-d}{a_{1}}<2(n+1) \frac{d}{a_{0}}
\end{array}
$$

[27 b]

Se si ammette un integrale particolare per l'onda incidente nella forma

$$
w_{1}=A(\omega) e^{i \omega\left(t+z_{!}^{\prime} a_{!}\right)}
$$

allora la teoria della riflessione dà le soluzioni particolari:

$$
\begin{aligned}
& w_{0}=\frac{2 p}{p+1} A(\omega) e^{i \omega\left(t+\frac{z-d}{a_{0}}+\frac{d}{c_{1}}\right)} \\
& \bar{w}_{0}=\frac{2 p}{p+1} A(\omega) e^{i(1)\left(t-\frac{z+d}{a_{0}}+\frac{d}{a_{1}}\right)}
\end{aligned}
$$




$$
\begin{aligned}
& \bar{w}_{1}=\frac{p-1}{p+1} A(\omega) e^{i(1)\left(t-\frac{z-2 d l}{a_{1}}\right)} \\
& w_{0}^{(v)}=\frac{2 p}{p+1}\left(\frac{1-p}{1+p}\right)^{v} A(\omega) e^{i \omega\left[t-\frac{z-(2 v+1) d}{a_{0}}+\frac{d}{a_{1}}\right]} \\
& \left.\bar{w}_{0}^{(v)}=\frac{2 p}{p+1}\left(\frac{1-p}{1+P}\right)^{v} A(\omega) e^{i \omega[t} \frac{z+(2 v+1) d}{a_{0}}+\frac{d}{a_{1}}\right] \\
& \bar{w}_{1}^{(v)}=\frac{4 p}{(1+p)^{2}}\left(\frac{1-p}{1+p}\right)^{v-1} A(\omega) e^{i(1)\left[t-\frac{z-2 d}{a_{1}}-\frac{2 v d}{a_{0}}\right]}
\end{aligned}
$$

L'onda $w_{1}$ deve essere supposta speciale come a pag. 7 .

$$
\begin{array}{lll}
w_{1}=0 & t<-\frac{z}{a_{1}}+\frac{d}{a_{1}} \\
w_{1}=e^{i \sigma\left(t+z / a_{1}\right)} & \text { per } & t \geq-\frac{z}{a_{i}}+\frac{d .}{a_{2}}
\end{array}
$$

allora si ha

$$
A((1))=\frac{1}{2 \pi i} \frac{e^{--i(1) d / a_{1}}}{(1)-\sigma}
$$

Nell'intervallo $(2 n+1) \frac{d}{a_{0}} \leq t<(2 n+3) \frac{d}{\|_{0}}$

si ottiene per $\varepsilon_{a}$ :

$$
\zeta_{0}=\frac{1}{2 \pi i} \frac{4 p}{1+p} \int_{-\infty}^{+\infty} \frac{\left(\frac{1-p}{1+p}\right)^{n+1} e^{-2 i(1)(n+1) d a_{0}}-1}{\frac{1-p}{1+p} e^{-2 i(1) d / a_{0}}-1} e^{i(1)\left(t-d / a_{n}\right)} \frac{d(1)}{(1)-\sigma} n \geq 1
$$

Per ralcolare questo integrale mediante integrazione per una via complessa lo si divide in due parti

$$
\tau_{n}=\frac{1}{2 \pi i} \frac{4 p}{p+1} \int_{-\infty}^{+\infty} \frac{\left(\frac{1-\boldsymbol{p}}{1+\boldsymbol{p}}\right)^{n+1}}{\frac{1-p}{1+p} e^{2 i(1) d / a} 0-1} e^{i(t)\left(t-(2 n+3) d / a_{11}\right)} \frac{d(1)}{\omega-\sigma}
$$




$$
-\frac{1}{2 \pi i}-\frac{4 p}{p+1} \int_{-\infty}^{+\infty} \frac{1}{\frac{1-p}{1+p} e^{-2 i \omega d / a_{0}-1}} e^{i(1)\left(t-d / a_{0}\right)} \frac{d \omega}{(1)-\sigma}
$$

Per $t<(2 n+3) d / a_{0}$ scompare il primo termine di [32'] $\tau_{j}$ viene dunque 1 appresentato nell'intervallo considerato solo col secondo termine, generalmente non più dipendente da $n$. Per calcolare questo secondo integrale, si devono calcolare anzitutto $i$ poli della frazione integranda. Oltre cle per $(1)-\sigma$ questi si trovano anche in

$$
\omega_{\mathbf{r}}=(2 r+1) \frac{a_{0}}{2 d} \pi+i \frac{a_{0}}{2 d} \ln \frac{p+1}{p-1}, \quad\left(r=0_{1}+1+2 \ldots . .\right)
$$

Se si integra ora intorno ai poli, si ottiene:

$$
\begin{gathered}
c_{0}=-\frac{4 p}{1+p} \frac{1}{\frac{1-p}{1+p} e^{-2 i u_{/}^{\prime} u_{0}}-1} e^{i \sigma\left(t-d / a_{0}\right)} \\
+i \frac{a_{0}}{d} \frac{2 p}{p-1} \sum_{r=-\infty}^{+\infty} \frac{1}{\left(0_{\mathrm{r}}-\sigma\right.} e^{i\left(1_{\mathrm{r}}\left(t+d / a_{0}\right)\right.}, \sigma \mp()_{\mathrm{r}}
\end{gathered}
$$

e questa soluzione vale per

$$
3 \frac{d}{a_{0}}<\iota<\infty
$$

L'equazione [32"] si deve completare con

e con

$$
\varsigma_{0}=0 \quad \text { per } \quad t<d / a_{0}
$$

$$
\zeta_{0}=\frac{4 p}{p+1} e^{i \sigma\left(t-d / a_{0}\right)} \quad \text { per } \quad d \leq t<\frac{3 d}{a_{0}}
$$

In modo del tutto analogo si può calcolare. l'onda $W_{1}$. Anzitutto si ottiene per $2 n d / a_{0} \leq t-\frac{z-d}{u_{1}}<2(n+1) d / a_{0}$ $W_{1}=\frac{1}{2 \pi i} i\left\{\frac{p-1}{p+1}+\frac{4 p}{(p+1)^{2}} \vee\left(\frac{1-p}{1+p}\right)^{v-1} e^{\left.-2 i(1) v d / a_{0}\right\}}\right\}^{i(1)}\left(t-\frac{z-d}{a_{1}}\right) \frac{d \omega}{(1)-\sigma}$ 
Od anche

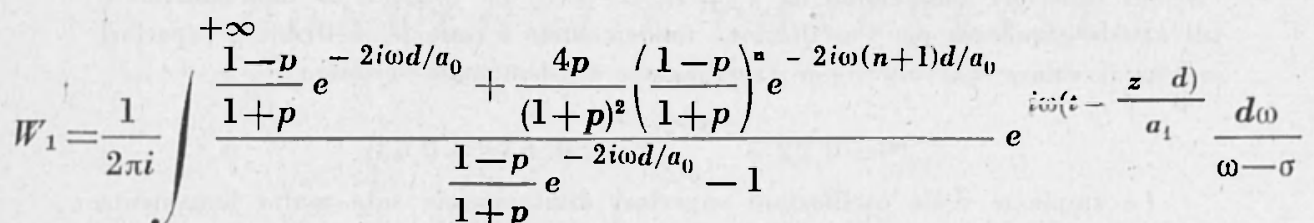

$$
\begin{aligned}
& -\infty
\end{aligned}
$$

Scindendo anche questo integrale come quello dell'equazione [32] in integrali parziali, l'integrale parziale, che corrisponde al $3^{\circ}$ sommante del numeratore in [35'], nell'integrale considerato è zero. I poli sono nuovamente dati da [33] o l'integrazione porta alla soluzione

$$
\begin{aligned}
& W_{1}=0 \quad, \quad \text { per } t<\frac{z-d}{a_{1}} \\
& W_{1}=\frac{p-1}{\mu+1} e^{i \sigma\left(t-\frac{z-d}{a_{1}}\right)} \quad \text { per } \frac{z-d}{a_{1}}<t<\frac{z-d}{a_{1}}+2 \frac{d}{a_{0}} \\
& W_{1}=\frac{\frac{1-p}{1+p}-e^{-2 i \sigma d / a_{0}}}{\frac{1-p}{1+p} e^{-2 i \sigma d / a_{0}}-1} e^{i-i i-\frac{z-d)}{a_{1}}} \\
& -i \frac{a}{d} 2 p \sum_{\mathrm{r}=-(1)}^{+\infty} \frac{1}{(1)_{0}-\sigma} e^{i \omega_{\mathrm{r}}\left(i-\frac{z-d}{a_{1}}+\frac{4 d}{a_{0}}\right)}, \text { per } \frac{z-d}{a_{1}}+2 \frac{d}{a_{0}} \leq t<\infty
\end{aligned}
$$

L'apparire delle frequenze proprio (1) e una conseguenza del fatto che sulla superficie limite $z=O$ e $z=d$ hanno periodicamente luogo nuove divisioni di energia. Il periodo fondamentale ̀̀ $T_{0}=4 d / a_{0}$. Le frequenze proprio devono essere complesse, perché l'energia disponibile non è infinitamente grande. Naturalmente si potrebbe rappresentare il fenomeno anche per mezzo di onde, nelle quali appaia la frequenza $\sigma$. $\mathrm{Ma}$ in una simile descrizione in ciascuno degli intervalli $(2 n+1) \frac{d}{a_{0}}<t<(2 n+3) \frac{d}{a_{0}}$ le ampiezze e le fasi sarebbero dipendenti da $n$. In questo cambiamento delle ampiezze e delle fasi con l'intervallo si esprime appunto l'apparire delle frequenze proprie.

Per ottenere una visione evidente di tali rapporti, si aggiungono qui alcuni calcoli numerici: come a pag. 11 sia $\sigma=1,51+1,07 i$ ed $a_{1}=1,6 \mathrm{~m} / \mathrm{ms}$. Per la velocità nello strato di materiale sciolto sia supposto $a_{0}=0,8 \mathrm{~m} / \mathrm{ms}$. Infine sia $d=2 \mathrm{~m}$ e $p=2$. Allora nell'intervallo $-\infty<t<2,5 \mathrm{~ms}$ l'oscillazione $\zeta_{0}=O$. Nell'intervallo $O \leq \tau=t-2,5<5 \mathrm{~ms}$ è $\varepsilon_{0}=2,67 \mathrm{e}^{-1,07 \tau} \sin 1,51 \tau$ nell'intervallo $O \leq \tau^{\prime}=t-7,5<\infty$ consta di una parte $\varepsilon_{v}(1)=1,84,10^{-4} e^{-1,07 \tau} \sin$ $\left(1,51 \tau^{\prime}+2,55\right)$ e di una parte $\varepsilon_{v}(2)$, che rappresenta una oscillazione smorzata 
periodica. 11 periodo fondimentale di $\zeta_{0}(*)$ ammonta a $10 \mathrm{~ms}$. I periodi delle oscillazioni superiori conseguono da $T_{r}=10 /(2 r+1)$. Lal costante di smorzamento ì di uguale grandezza per loscillazione fondamentale a tutte le oscillazioni superiori ed hat il valore $0,22 \mathrm{~ms}^{-1}$. l'er l'oscillazione fondamentale si ottiene:

$$
i_{00}^{(2)}=0,22 e^{-0,22 \tau^{\circ}} \cos \left(0,63 \tau^{\prime}-0,63\right)
$$

Le ampiezze delle oscillazioni superiori diminuiscono solo molto lentamente crescendo $r$, cosicché le frequenze più alte influiscono ancora notevolmente sul quadro dell'oscillazione. $\dot{E}$ importinte, che lia parte $r_{v}^{(i)}$ retroceda di fronte a $\zeta_{0}(2)$. Dopo il passiggio del primo impulso nei primi $5 \mathrm{~ms}$ le frequenze proprie si fanno fortemente sentire, tanto più che la loro parte immaginaria è notevolmente inferiore di quelli di $\sigma$. Nello spettro compariscono quindi frequenze più basse.

Del tutto simili sono le condizioni per $W_{1}$. Appariscono le stesse frequenze proprie come $\tau_{0} \cdot W_{1}$ è zero per $t<\frac{z-l}{a_{1}}$.

Nell'intervallo $\frac{z-d}{a_{1}}=.-\frac{z-d}{a_{1}}+5$ ms si ottiene: $W_{1}=0,33$ e $1,07\left(t \quad \frac{z-d}{a_{1}}\right)$ $\sin .1,51\left(t-\frac{z-d}{a_{1}}\right)$

Nellintervallo $\frac{z-d}{a_{1}}-\imath=\imath-5<x$ la prima parte di $W_{1}$ ammonta a

$$
W_{1}^{(1)}=1,44.10^{-2} e^{-1.07}\left(t-\frac{z-d}{a_{1}}\right) \sin \left[1,51\left(\tau-\frac{z-d}{a_{1}}\right)+1,28\right]
$$

Nella seconda parte il contributo dellonda col periodo fondamentale importa:

$$
W_{10}^{(2)}=-0,07 e^{-0,22\left(\tau-\frac{z-d}{a_{1}}\right)} \cos \left[0,63\left(\tau-\frac{z-d}{a_{1}}\right)-0,63\right]
$$

Si vede che per $W_{1}$ le frequenze proprie non hanno una parte importante come per $C_{0}$, ma anche per l'onda riflettente sulla superficie terrestre appaiono nello spettro frequenze più basse che nel caso dell'onda incidente.

Pare che l'effetto di interferenza discusso a pag. 1 operando insieme all'effetto, discusso per ultimo, della superficie superiore di un mezzo stratificato determini sostinzialmente lo spettro delle onde sismiche da esplosione. Anche per le onde naturali da terremoto i procedimenti dovuti all'incidenzal allia superficie superiore degli strati esterni della terra, dovrebbero essere di importanzia per lo spettro di queste onde. Si richiede naturalmente di estendere la ricerca al caso di incidenza non perpendicolire.

\section{RIASSUNTO}

Viene discusso l'effetto dinterferenza, che provoca la variazione dello spettro delle onde sismiche generate da esplosioni in un mezzo elastico ideale.

L'influsso della così detta .Verwitterungszone. (zona in cui originano le onde per urto) sembra essere predominante. 